\title{
BMJ Open Improving the HIV PrEP continuum of care using an intervention for healthcare providers: a stepped-wedge study protocol
}

\author{
Kristin Ming (D) , ${ }^{1}$ Isha Shrestha, ${ }^{1}$ Alexander Vazquez, ${ }^{1}$ James Wendelborn, ${ }^{1}$ \\ Veronica Jimenez, ${ }^{1}$ Nadra Lisha, ${ }^{1}$ Torsten B Neilands, ${ }^{1}$ Hyman Scott, ${ }^{2}$ Albert Liu, ${ }^{2}$ \\ Wayne Steward, ${ }^{1}$ Mallory O Johnson, ${ }^{1}$ Parya Saberi (D) ${ }^{1}$
}

To cite: Ming K, Shrestha I, Vazquez A, et al. Improving the HIV PrEP continuum of care using an intervention for healthcare providers: a stepped-wedge study protocol. BMJ Open 2020;10:e040734. doi:10.1136/ bmjopen-2020-040734

- Prepublication history for this paper is available online. To view these files, please visit the journal online (http://dx.doi. org/10.1136/bmjopen-2020040734).

KM and IS contributed equally.

Received 20 May 2020 Revised 02 June 2020 Accepted 03 June 2020

A) Check for updates

(C) Author(s) (or their employer(s)) 2020. Re-use permitted under CC BY-NC. No commercial re-use. See rights and permissions. Published by BMJ.

${ }^{1}$ Department of Medicine, Center for AIDS Prevention Studies, University of California, San Francisco, San Francisco, California, USA

${ }^{2}$ Bridge HIV, San Francisco Department of Public Health,

San Francisco, California, USA

Correspondence to

Professor Parya Saberi;

Parya.Saberi@ucsf.edu

\section{ABSTRACT}

Introduction Pre-exposure prophylaxis (PrEP) has demonstrated to be a highly effective method for preventing HIV; however, many individuals with PrEP indications are not receiving PrEP. Primary care settings provide an opportunity to offer PrEP to a wide range of patients. In this paper, we describe the PrEP Optimisation Intervention (PrEP-OI), which includes a PrEP Coordinator and a web-based panel management tool (called PrEP-Rx), and is targeted at healthcare providers (HCPs) to increase PrEP uptake and persistence among those at risk for acquiring HIV.

Methods and analysis The PrEP-Ol study evaluates the efficacy of the PrEP intervention (PrEP Coordinator + PrEP-

$\mathrm{Rx}$ ) to increase PrEP prescriptions through a stepped-wedge design among 10 primary care clinical sites in the San Francisco Department of Public Health. The number of PrEP initiation prescriptions constitute the primary outcome, and we hypothesise that the mean number of PrEP prescriptions written will significantly increase after the clinics initiate PrEP-Ol versus before this intervention. Secondary objectives include: 1-differences in PrEP initiation, duration of use and reasons for discontinuation based on patient's age, race/ethnicity and sex/gender, and by clinic and HCP characteristics, 2-sustainability of the intervention during a 12-month follow-up after the stepped-wedge phase, and 3 -facilitators and barriers of PrEP delivery and experiences with the proposed PrEP intervention through qualitative interviews with HCPs. The results of this study can provide valuable insight into methods to reduce the burden of PrEP care on HCPs and improve PrEP continuum of care.

Ethics and dissemination This study and its protocols have been approved by the University of California, San Francisco (UCSF) Institutional Review Board. Study staff will disseminate findings locally (eg, the UCSF Centre for AIDS Prevention Studies' Community Engagement Core), statewide (eg, the California Department of Public Health's Office of AIDS) and nationally and internationally at conferences related to HIV.

Trial registration number NCT03532191.

\section{BACKGROUND}

Pre-exposure prophylaxis (PrEP) has been shown to be highly effective for the
Strengths and limitations of this study

- All of the clinics in this study were primary care clinics, which facilitated linkage to care when there was a need for a follow-up.

- The use of a pre-exposure prophylaxis (PrEP) Coordinator in managing a panel of PrEP patients may improve the number of PrEP initiations, adherence, persistence and provision of clinical care, and reduce the time burden on the healthcare provider.

- The web-based panel management tool's (PrEP-Rx) tailored features help the PrEP Coordinators to efficiently manage a patient's PrEP continuum of care and included an HIV Risk Assessment Questionnaire to assess and capture a patient's HIV risk.

- All of the clinical sites in this study were primary care clinics located in San Francisco and the data from this study may not be generalisable to other non-primary care clinics and to primary care clinics outside of San Francisco.

- PrEP-Rx is not currently integrated with electronic health records, and some healthcare providers may be reluctant to log into additional system due to time constraints.

prevention of HIV in randomised clinical trials, ${ }^{1-6}$ demonstration projects ${ }^{7-9}$ and clinical settings, ${ }^{10}$ but PrEP uptake in the USA has not been commensurate with the need. ${ }^{11-13}$ Despite data indicating approximately 220000-225000 individuals currently on PrEP as of May 2020, ${ }^{14}$ the Centers for Disease Control and Prevention (CDC) estimates that there are over 1.2 million adults with a PrEP indication. ${ }^{15}$ Therefore, only $18 \%$ of these individuals are currently receiving PrEP. Different models of improving PrEP uptake and persistence have been evaluated, including through health maintenance organisations, sexually transmitted infection (STI) clinics and primary care practice settings. ${ }^{16}$ Each has their strengths and weaknesses. 
Primary care settings provide the opportunity to offer PrEP as part of routine medical care, reach a broader patient population who may not use STI clinics or be aware of their exposure to HIV, and integrate STI, HIV and creatinine testing needed for PrEP continuation into existing health structures. However, some of the barriers in primary care settings may be that healthcare providers (HCPs) lack knowledge and willingness to prescribe PrEP, ${ }^{11} 131718$ may not be routinely assessing HIV risk and may not be prepared to provide risk-reduction or adherence support. ${ }^{16}$ For example, in a 2015 national survey of primary care providers (PCPs), only $66 \%$ were aware of PrEP. ${ }^{19}$ However, once PrEP was defined, $91 \%$ indicated a willingness to prescribe it for high-risk patients and expressed an interest in education about how to deliver PrEP. ${ }^{19}$

A descriptive report on the early experiences with PrEP uptake and persistence in San Francisco identified the following priority steps for HCPs to increase PrEP use and maximise public health impact: (1) increase PrEP knowledge among HCPs and (2) expand PrEP access by training HCPs and developing tools to facilitate PrEP delivery in clinical settings. ${ }^{20}$ Additionally, based on the framework of the PrEP care continuum, interventions to enhance PrEP uptake include HCP education, tools to assess sexual risk and systems that minimise HCP burden. ${ }^{21}$ Given these proposals and recommendations, innovative and effective approaches are needed to support and provide guidance to HCPs regardless of level of experience prescribing PrEP.

Therefore, we propose the PrEP-Optimisation Intervention (PrEP-OI) targeted at HCPs to increase PrEP uptake and persistence among those at risk for HIV acquisition. This intervention includes two primary components: (1) centralised PrEP coordination overseen by a PrEP Coordinator (a non-clinical support staff member) who can support multiple HCPs. PrEP Coordinators follow-up on referrals from HCPs for PrEP initiations, support continuity of care for existing PrEP patients and outreach to patients who may benefit from PrEP by reviewing registries for STIs; and (2) an integrated web-based panel management tool called PrEP-Rx, which includes a structured HIV Risk Assessment, a Patient Timeline, Coordinator's Dashboard, and Provider's Dashboard (defined in Intervention section). PrEP Coordinators use this tool to assess patients' HIV risk and manage patients' timeline of PrEP use. This intervention was previously pilot tested at one San Francisco Department of Public Health (SFDPH) clinic, and was shown to be acceptable and feasible to HCPs. ${ }^{22}$ In the PrEP-OI study, we hypothesise that the mean number of prescriptions issued will be significantly higher after the clinics initiate the PrEP intervention versus before this intervention. In this paper, we describe the intervention in detail and our plans to evaluate the efficacy of PrEP-OI (PrEP Coordinator + PrEP-Rx) to increase PrEP initiation prescriptions through a steppedwedge design among primary care clinical sites in the SFDPH safety-net system.
PREP-OI INTERVENTION

\section{The PrEP Coordinator}

The PrEP Coordinator is a designated non-clinical support staff person who coordinates care between patients and HCPs. The PrEP Coordinator uses PrEP-Rx to conduct or augment the PrEP follow-up conducted by HCPs and perform panel management activities. The role of the PrEP Coordinator is to facilitate new PrEP initiations and support continued care engagement for existing PrEP patients. The PrEP Coordinator reaches out to patients, evaluates HIV risk and desire to initiate PrEP, counsels and educates patients on the use of PrEP and HIV risk reduction, orders and monitors necessary laboratory tests for PrEP initiation and follow-up (using standing order protocols), educates patients on the self-swabbing for STIs (as needed) and assesses medical insurance coverage for PrEP. Once the patient is ready to initiate PrEP, the PrEP Coordinator initiates and routes the prescription order to the HCP for approval, verifies PrEP prescription pick-up and initiation, and conducts outreach at 1 week and 1 month to provide medication adherence counselling and inquire about potential side effects. The PrEP Coordinator follows up with the patient every 3 months and assesses for adherence, screens for STIs and schedules laboratory visits as indicated by the CDC guidelines. ${ }^{23}$ Additionally, the PrEP Coordinator communicates with HCPs to address any clinical health concerns, including initiation of postexposure prophylaxis (PEP) or a switch from PEP to PrEP, and provides updates on new PrEP discoveries to HCPs. Here, we detail the PrEP Coordinator training and workflow.

\section{PrEP Coordinator training}

PrEP Coordinators receive comprehensive training prior to being placed at clinical sites. Training includes the following (15-48 hours).

\section{PrEP basics}

PrEP Coordinators are trained on the 'PrEP Basics' which includes how to effectively conduct tailored one-on-one patient counselling according to the patient's existing PrEP knowledge and how PrEP may fit within their daily lives. This training consists of details of PrEP dosing with tenofovir disoproxil/emtricitabine (FTC), the time it takes to reach protective levels, potential side effects, importance of PrEP adherence, steps to be followed if a dose is missed or the medication is discontinued, obtaining refills and stopping/restarting PrEP. PrEP Coordinators also receive training on PEP and STI selfswabbing. Depending on the PrEP Coordinator's prior PrEP knowledge and experience, this training may take anywhere from 2 to 8 hours.

\section{Ordering laboratory tests}

PrEP Coordinators are trained to adhere to the health system guidelines regarding laboratory testing for PrEP initiation and quarterly follow-up (these guidelines are based on CDC guidance). They receive training on the 
importance of each laboratory test to ensure that the tests are appropriately ordered (using standing order protocols) and to answer any questions patients may have regarding the tests ordered (1-4hours).

\section{Patient education on STI self-swabbing}

PrEP Coordinators learn how to educate patients on self-swabbing methods of oral and rectal swabs. Written instructions on self-swabbing (developed in English, Spanish and Chinese) or instructional videos produced in collaboration between SFDPH and Impulse SF on YouTube are available to facilitate this process (1-2 hours) .

\section{Insurance navigation}

PrEP Coordinators receive training on local insurance and coverage programmes; those working in youth clinics receive additional training on adolescent and young adult considerations specific for PrEP (eg, minor consent for sexual health). In addition, PrEP Coordinators learn how to submit applications to the current PrEP manufacturer's Patient Assistance Programme and Coupon Co-Pay programme. Ensuring PrEP Coordinators have knowledge of local resources allows them to support the patient's individualised needs (1-6 hours).

\section{Shadowing}

Prior to starting their work at the clinical sites, PrEP Coordinators shadow other PrEP Coordinators/Navigators in the community. During this time, they are able to observe practices of shared decision making, adherence counselling and motivational interviewing, and other patientcentred skills. Shadowing also allows PrEP Coordinators to observe how PrEP services are implemented and gain a better understanding of the PrEP workflow of a clinic (4-8 hours).

\section{Role-playing}

Role-playing allows PrEP Coordinators to address topics that generally come up during conversations with patients. This is especially important for PrEP Coordinators who have had less direct service experience with patients. When role-playing, it is best to have a group of three or more PrEP Coordinators. One person plays the role of the patient, another is the PrEP Coordinator, and the third person can be the observer who provides feedback on the interaction (2-4hours).

\section{Charting in the electronic health record}

PrEP Coordinators use an electronic health record (EHR) to complete their work. PrEP Coordinators receive training on how to schedule appointments, document notes, order laboratory tests, send prescription orders to the HCP and understand the channels for communicating with other HCPs and clinical staff. Depending on the PrEP Coordinator's previous knowledge and experience, and depending on the user friendliness of the EHR, this training could take anywhere between 4 and 16 hours.

\section{Ongoing supervision}

Once PrEP Coordinators begin their work at the clinical sites, they meet weekly with PrEP-experienced clinicians to discuss pertinent clinical cases. This provides an opportunity for Coordinators to receive support from each other and from the clinicians, and problem-solve issues that come up in their respective clinics. These meetings are also a chance to receive ongoing training on PrEP updates, such as 2-1-1 PrEP (also known as On-demand or Event-based PrEP dosing) ${ }^{5}$ and tenofovir alafenamide/ FTC (TAF/FTC) for PrEP. ${ }^{24}$ Training includes the understanding of new regimens, comparing them to the existing PrEP regimen, tools to help educate HCPs and patients, and how to help patients determine which regimens may be best suited for them.

\section{PrEP Coordinator workflow}

The PrEP Coordinator patient panel can be created using two sources.

\section{Existing patients}

Prior to starting at a clinical site, the PrEP Coordinator receives an internal list of existing PrEP patients from the clinic. The PrEP Coordinator reviews the patients' charts and adds them into the PrEP-Rx panel management tool to begin tracking them for monitoring and follow-up. With the HCP's permission, the PrEP Coordinator reaches out to the patients to introduce themselves and let the patient know how to contact them if they have any questions about their PrEP care. In cases where the patients are behind in completing quarterly PrEP laboratory follow-up, the PrEP Coordinator sends a secure message to the HCP to check in about this patient and request permission to reach out to the patient and schedule them for laboratory follow-up and future appointments. On contacting the patient, the PrEP Coordinator then documents each interaction in the EHR. PrEP Coordinators make a minimum of three outreach attempts to each patient. There may be instances where the HCP chooses to opt out of having a PrEP Coordinator involved in the patient's care. In these cases, the PrEP Coordinator documents that they are not providing panel management for this patient in the patient's chart.

\section{New referrals}

PrEP Coordinators can receive PrEP referrals from HCPs, other clinical and social services staff, self-referral from patients and STI registries. The PrEP Coordinator may also ask clinic staff to inform the patient about PrEP services when they present for STI treatment. If a patient expresses interest, the PrEP Coordinator reaches out to the patient to initiate PrEP. Coordinators attend clinical huddles (brief daily meetings in which a care team reviews their patient list for that day at the beginning of each shift) at each site and periodically attend provider meetings to embed themselves into the clinic's landscape. This can help facilitate better communication between the PrEP Coordinator and the clinic staff and can aid in increasing new PrEP referrals. 
When the PrEP Coordinator receives a new referral from a HCP, the PrEP Coordinator contacts the patient a minimum of three times. If the Coordinator does not make successful contact with the patient after three attempts, they send a message notifying the patient's HCP. If the PrEP Coordinator is able to reach the patient, and the patient confirms their interest in starting PrEP, the PrEP Coordinator and patient meet in person or over the phone to complete an initial patient consultation and HIV Risk Assessment using PrEP-Rx. The PrEP Coordinator then orders the appropriate PrEP Initiation laboratory tests. After the patient completes these tests, the PrEP Coordinator sends the prescription request to the HCP, who approves the medication after reviewing laboratory results and sends the prescription to the patient's preferred pharmacy. This model could support same day PrEP starts. The PrEP Coordinator follows up with the patient the next day to ensure PrEP pick up and initiation. If unable to reach the patient, the PrEP Coordinator can call the pharmacy to confirm if patient picked up their prescription. One week after initiation, the PrEP Coordinator calls the patient to assess how the patient is doing with the new PrEP regimen and to discuss any side effects. One month after initiation, the PrEP Coordinator follows up with the patient (in-person or by phone) to assess acute HIV and STI symptoms, review side effects and adherence, and make an appointment with the HCP and/or for a repeat HIV laboratory test, if necessary. At each check in, the PrEP Coordinator inquires about how the patient is doing with taking PrEP and answers any questions the patient may have about their PrEP use or refers the patient to their HCP for follow-up.

PrEP Coordinators contact patients at least 10 days prior to when they are due for quarterly PrEP laboratory tests and schedule patients for a laboratory appointment and/ or a follow-up appointment with the PrEP Coordinator at the clinic. Whether the follow-up takes place in person or over the phone, the PrEP Coordinator completes the 'Follow-Up Consultation' section of PrEP-Rx to guide discussion at each follow-up.

\section{PrEP-Rx}

PrEP-Rx is a web-based panel management tool originally developed as part of a pilot study ${ }^{22}$ that contains three main functions: (1) a comprehensive self-administered HIV risk assessment using an integrated survey tool; (2) automated reminders to PrEP Coordinators for patient lab monitoring and follow-up visits for adherence, side effect assessment and risk reduction counselling; and (3) a PrEP timeline for each patient to allow PrEP Coordinators and HCPs to see a patient's PrEP use history and upcoming visits in one snapshot. Additionally, our website (www.preprx.ucsf.edu) contains information about the study, patient resources and handouts (eg, self-swabbing instructions, PrEP Basics, etc), PrEP updates and educational material for HCPs (eg, guidelines, publications, conference proceedings, etc), and PrEP-Rx login link. PrEP-Rx was created using a Health Insurance Portability

\begin{tabular}{ll}
\hline $\begin{array}{l}\text { Table 1 } \\
\text { Patient } \\
\text { status }\end{array}$ & PrEP-Rx patient status bar categories \\
\hline $\begin{array}{l}\text { Referred } \\
\text { Outreach }\end{array}$ & $\begin{array}{l}\text { New referral or new patient added. } \\
\text { In the process of outreaching to a new } \\
\text { patient, but haven't reached them yet. }\end{array}$ \\
Active & $\begin{array}{l}\text { Patient is engaged, on PrEP or planning to } \\
\text { start PrEP. }\end{array}$ \\
$\begin{array}{l}\text { Needs } \\
\text { support }\end{array}$ & $\begin{array}{l}\text { Patient's PrEP care is on hold and needs } \\
\text { support. }\end{array}$ \\
$\begin{array}{l}\text { Lost to follow- } \\
\text { up (LTFU) }\end{array}$ & $\begin{array}{l}\text { Patient has been unresponsive or difficult to } \\
\text { reach. }\end{array}$ \\
Inactive & $\begin{array}{l}\text { No longer tracking this patient (eg, not } \\
\text { interested in PrEP, discontinued PrEP or has }\end{array}$ \\
& been LTFU for extended period).
\end{tabular}

PrEP, pre-exposure prophylaxis .

and Accountability Act (HIPAA) compliant Salesforce backend and a Qualtrics survey was used to assess risk among potential PrEP users.

PrEP-Rx contains four main components: (1) patient registry, (2) PrEP Coordinator's dashboard, (3) provider's dashboard and (4) reports.

\section{Patient registry}

This includes all the patient-related features needed to manage a patient's PrEP use. The PrEP Coordinator uses the patient registry along with the Coordinator's dashboard to manage their PrEP panel. The patient registry includes the following sections.

\section{Patient details}

This section includes patient demographic information (eg, name, date of birth, medical record number (MRN), sex at birth, gender identity, race/ethnicity), primary language, PCP, primary care clinic, contact information, preferred pharmacy, insurance/coverage programme used to access PrEP and assigned PrEP Coordinator. Patient details also include the 'Patient Status Bar,' which is helpful in determining where the patient stands in their PrEP use (see table 1).

\section{Risk assessment}

The Risk Assessment Questionnaire was created by the study team using the CDC risk index ${ }^{23}$ input from behavioural risk assessment experts at the University of California, San Francisco (UCSF) Center for AIDS Prevention Studies for questions on transgender spectrum individuals, risk assessment questions for heterosexual risk from the CDC guidelines ${ }^{23}$ and information from a publication by Smith $e t a l^{25}$ on risk associated with injection drug use. We modified this tool to include three tiers of risk: low, medium and high. Details of this risk assessments have previously been published.$^{22}$ This questionnaire is in Qualtrics and responses are integrated with Salesforce. The patient completes the Risk Assessment on 
a tablet during the PrEP Initiation Visit with the PrEP Coordinator. If initiation is being completed over the phone, the PrEP Coordinator emails the unique Qualtrics survey link to the patient and they complete it on their mobile device or the PrEP Coordinator reads the questions to the patient and completes the survey. The risk assessment is available in English, Spanish and Chinese.

\section{Patient timeline}

When a patient is prescribed PrEP, the PrEP Coordinator initiates the patient's PrEP timeline, which auto-generates a list of standardised follow-up events with calculated target dates, which include PrEP picked up ( $\mathrm{x}$ days after PrEP prescription date), PrEP initiation ( $\mathrm{x}$ days after PrEP prescription date), 1-week follow-up, 1-month follow-up and quarterly follow ups. PrEP-Rx emails reminders to the PrEP Coordinator of upcoming incomplete events. When the event is completed, the PrEP Coordinator updates the event with the date completed, which automatically shifts future event dates to maintain the predetermined time frame between events (eg, if a quarterly laboratory test is completed 2 weeks after the initial target date, all future quarterly laboratory test dates are subsequently moved back by 2 weeks). PrEP Coordinators can also create ad hoc events, for example, an event to remind them to order an annual Hepatitis C laboratory test.

\section{Patient consultations}

There are two kinds of patient consultations: initial and follow-up. The questions in each of these consultations were developed through discussion with clinicians who are coinvestigators for this study. Topics include assessing for acute HIV symptoms, screening for STIs, evaluating medication adherence and reviewing need for refills. Medication adherence is evaluated based on the questions: Rate your ability to take your medication as directed by your HCP in the last 30 days (response options range from 'excellent' to 'very poor') ${ }^{26}$ and How many pills would you estimate you have taken in the last 30 days? ${ }^{27}$ The initial consultation guides the PrEP counselling visit and should be completed prior to the patient starting PrEP. The follow-up consultation should be completed at each quarterly visit. The information collected at each consultation can be exported as text, which can then be copied and pasted into the EHR.

\section{PrEP Coordinator's dashboard}

This dashboard provides a tracking method for all upcoming incomplete events associated with 'active' (see table 1) patients on the PrEP Coordinator's panel. It is sorted by the due date so that upcoming events are at the top. Overdue events appear in red and completed events disappear from the dashboard.

\section{Provider's dashboard}

This dashboard summarises the patient's pertinent PrEP information in one simple format. It consists of four components for each patient: (1) Demographics (including patient's name, MRN, date of birth and phone number); (2) PrEP Timeline(s) (summary of current and prior PrEP use, including dates of follow-up appointments, laboratory visits, PrEP adherence and reasons for prior PrEP discontinuations, if applicable); (3) Risk categories (summary of risk and the risk assessment categories) and (4) Laboratory test results (including baseline and the last two laboratory results on file).

\section{Reports}

PrEP-Rx has the ability to generate reports, which can help PrEP Coordinators improve panel management. For example, a report with patients' past activities may help the PrEP Coordinator ensure these patients have a plan of action moving forward. A Clinic Snapshot report (which provides an overview of all the patients a PrEP Coordinator manages at each clinic) can be used to provide updates to clinics on the number of new PrEP starts and the total number of patients on PrEP at each clinical site.

\section{STUDY METHODS \\ Design}

We will evaluate the efficacy of the PrEP-OI (PrEP Coordinator + PrEP-Rx) to increase PrEP prescriptions through a stepped-wedge design among 10 primary care clinical sites in the SFDPH safety-net system. A stepped-wedge design is a type of one-way cross-over design in which all clinical sites begin the study without the intervention, and one-by-one initiate the intervention every month, with the order of site crossover determined at random to maximise internal validity. Compared with a traditional parallel-arm randomised controlled trial (RCT) design, the stepped-wedge brings intervention exposure to all clinical sites more rapidly and is more feasibly implemented because one site initiates the PrEP intervention every month, allowing the study team sufficient time to address any implementation questions on a per-clinical site basis. Because these considerations are critical in the proposed study, the stepped-wedge design was chosen. A disadvantage of the stepped-wedge design is that there is a systematic difference in calendar times between the treatment and control arms, making this design more vulnerable to the effects of secular trends than the standard parallelarms RCT. To address this concern, as recommended by Hayes and Moulton, ${ }^{28}$ our proposed analyses incorporates 3 months of prerandomisation data collection, our statistical models includes time trends, and we will explore pairwise comparisons of intervention-exposed and unexposed clinical sites at each monthly time point. Therefore, the PrEP-OI study has three phases including the prerandomisation phase, the stepped-wedge phase and the follow-up phase (see table 2).

For prerandomisation data collection, we are collecting data on those receiving PrEP in one of the study clinics during the 3 months prior to the stepped-wedge phase (ie, August-October 2018). For the patients on PrEP during this timeframe, EHR data are extracted for the time period starting 6 months prior to their most recent PrEP 
Table 2 Prerandomisation, stepped-wedge and follow-up phases

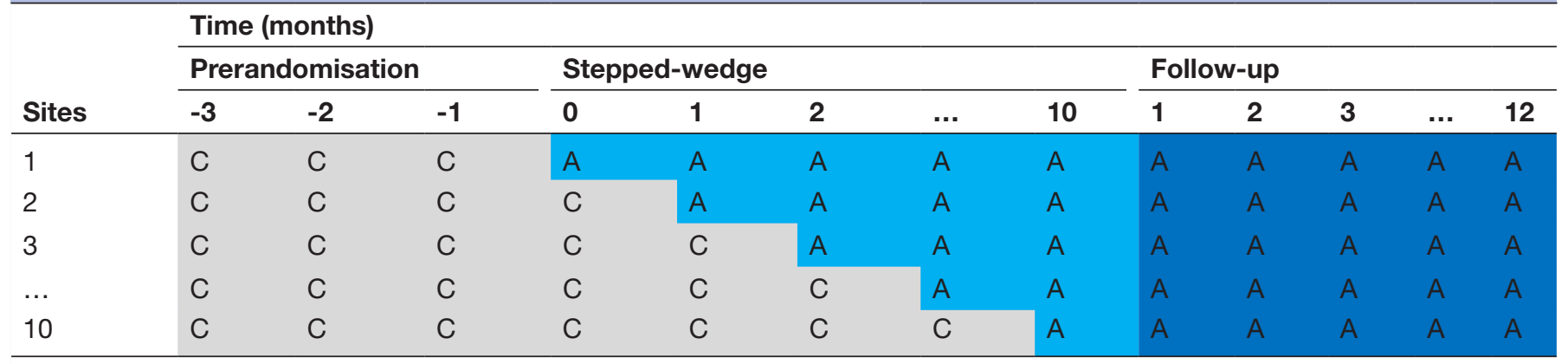

$\mathrm{A}$, intervention; $\mathrm{C}$, control.

start date, to capture any preliminary PrEP discussions between the HCP and the patient prior to prescribing PrEP. Patient data continue to be collected through the stepped-wedge phase, with patient data from clinics not yet randomised to initiate the intervention serving as control data.

All study procedures were reviewed and approved by the UCSF Institutional Review Board.

\section{Setting}

The 10 clinical sites consist of 12 primary care clinics part of the SFDPH large safety-net system that are spread over a wide geographical area in San Francisco and include a diverse patient population. Three clinics under one administration with overlapping HCPs and drop-in services for adolescents and young adults were grouped together for a total of 10 clinical sites. The study clinics were chosen based on their current involvement in PrEP initiatives for San Francisco residents and their willingness to participate.

\section{Participants}

The primary targets of the PrEP-OI study are HCPs employed at the study clinics who are able to prescribe PrEP. Patient data will also be analysed from patients who are seen at these clinics by the participating HCPs and who have been offered PrEP during the study.

\section{Sample size justification}

Power analyses were generated using the user-written Stata command-stepped-wedge-to compute minimum detectable effect sizes for the primary analysis to address the primary hypothesis. The study begins with 10 clinical sites. Since the intrasite correlation of PrEP prescribing is unknown, we considered low and high values of the intracluster correlation $=0.01-0.10$, respectively. ${ }^{29}$ Assuming $\alpha=0.05$, power $=0.80$ and 5 HCPs per clinical site, we computed the standardised minimum detectable mean difference $d$. Under these assumptions, we found $\mathrm{d}$ ranged from 0.25 to 0.28 . These effect size estimates for our primary hypothesis are between the thresholds of 0.20-0.50 for small and medium standardised effect sizes, ${ }^{30}$ suggesting that our proposed primary analysis has sufficient power to detect a small to medium effect.

\section{Clinic recruitment}

Prior to study launch, the study team met with each clinic's medical director to confirm interest in participating and gather clinic background information such as patient panel size, ratio of ancillary staff to HCPs, current PrEP practices and initial thoughts on how the intervention may fit seamlessly into the current clinic workflow. We then attended HCP meetings to present the proposed workflow, obtain buy-in and hear feedback and potential barriers that should be addressed early on. These meetings ensured we gained an understanding of each clinic's current practices and challenges around prescribing PrEP, and became familiar with the HCPs and key contacts.

\section{Randomisation procedures}

After the list of participating clinics was finalised, the order of clinical site onboarding was randomly selected as recommended by Hussey and Hughes ${ }^{31}$ to optimise the design to maximise power for hypothesis testing. All clinical sites began the study without the PrEP intervention, and then were randomised one-by-one on a monthly basis to begin receiving the intervention until all clinical sites were receiving the intervention. Clinics were notified of their planned start date 2 months in advance.

\section{Stepped-wedge phase and onboarding procedures}

In the stepped-wedge phase, a new clinical site began receiving the PrEP intervention each month from November 2018 to September 2019. Preparing for clinic onboarding began 2 months prior to each clinic's planned start date. During this period, a PrEP Coordinator was assigned to the clinic based on the clinic's proximity to other locations the PrEP Coordinator was working at and the clinic's volume of PrEP prescriptions. The study team met with the medical director to refine clinic-specific workflows, decide on the PrEP Coordinator's schedule based on availability of space and laboratory services, and agree on key contacts and preferred communication method for logistical and/or clinical questions. We also presented at a clinic all-staff meeting to provide training on PrEP and STIs, give an overview of the study, introduce the PrEP Coordinator, and review the services that the PrEP Coordinator could offer and how to refer patients. The 
PrEP Coordinator began reviewing existing patients on PrEP and adding them into PrEP-Rx to assess if patients were due for laboratory tests or needed support in their continuation of PrEP use.

\section{Follow-up phase}

Following the stepped-wedge phase, we are conducting a follow-up phase for 12 months (October 2019-September 2020) during which we will examine the continued use of the PrEP intervention at the study clinics to assess longterm use after HCPs have become increasingly familiar and comfortable with it. Therefore, in total, each clinic will receive the intervention for 13-23 months depending on their order of randomisation.

\section{Patient and public involvement}

PrEP-Rx was initially developed and shown to be acceptable and feasible among six HCPs in a pilot study conducted at a clinic in San Francisco. ${ }^{22}$ As part of this formative research, HCPs provided feedback on refinements to the design and function of PrEP-Rx through one-on-one qualitative interviews. This feedback served as a basis for the development of the newer version of PrEP-Rx used in this study. Additionally, PrEP Coordinators hired for this study were trained on PrEP-Rx and gave their input into how to make the tool most useful for their work, including adding the PrEP Coordinator's Dashboard and streamlining a way to export notes that could be copied into the EHR. PrEP-Rx was developed using an agile methodology, which involves incremental, iterative cycles of development that adjust to changing requirements dynamically. ${ }^{32}$ This gave the PrEP Coordinators multiple opportunities to test and refine features as they were built.

At the start of this study, we met with each clinic's medical director, which provided insight into how a PrEP Coordinator could fit into their existing PrEP workflow, any unique aspects to their clinic that should be considered, and suggestions on how best to train providers on PrEP and the referral process to the PrEP Coordinators. During weekly team meetings, PrEP Coordinators have an opportunity to bring up barriers or suggestions they are facing at their clinics to problem-solve as a group and standardise solutions across the clinics when possible. Finally, during the follow-up phase, we will conduct oneon-one qualitative interviews with HCPs and clinic staff to further get their feedback on the intervention and ways to make improvements for the remainder of the study.

\section{DATA COLLECTION AND MANAGEMENT PROCEDURES Sources of data}

We have four major sources of data: 1-patient data from EHR; 2-risk assessment survey; 3-HCP quantitative survey and 4-HCP qualitative interviews. For patients who have a PrEP prescription during the prerandomisation, steppedwedge or follow-up phases, we extract data from the EHR to assess adherence to CDC PrEP guidelines (ie, frequency of laboratory monitoring, adherence and risk reduction counselling, and follow-up) and HCPs' general PrEP prescribing practices (ie, methods of HIV risk assessment, systematic review of patients' STIs to evaluate HIV risk, number of refills at the first visit, and follow-up to establish PrEP initiation).

The patient's risk assessment survey is completed at PrEP initiation counselling to facilitate the conversation about whether to initiate PrEP. The survey provides data on the patient's level of HIV risk (high, medium or low) and PrEP indication (men who have sex with men, people who inject drugs) or people who have heterosexual sex). If the survey is unable to be completed (eg, the visit is conducted by phone and the patient does not have time or internet access), the PrEP Coordinator briefly screens the patient for PrEP indications and document this in their medical record.

The HCP quantitative survey is completed by HCPs during clinic onboarding. Finally, the one-on-one HCP qualitative interviews are conducted during the study's follow-up phase to examine facilitators and barriers of PrEP delivery, how the PrEP-OI services are working for the HCPs, and how these services can be improved to increase PrEP-prescribing practices. A purposive sampling approach ${ }^{33}$ guides selection of HCPs for interviewing, with a goal of having variability in participants' professional training (eg, physician, nurse practitioner), clinic where employed, role within clinic (eg, management responsibilities in addition to serving as a HCP) and degree of engagement with the PrEP Coordinator. Interviews are conducted by two project investigators (PS and WS) after receipt of verbal consent using a HIPAAcompliant web-based video conferencing platform. To facilitate an open discussion, team members serving as PrEP Coordinators are not involved in the interviews or data analysis. Each interview takes approximately 1 hour and participants are offered a US $\$ 50$ Amazon e-gift card. With participant permission, the interview is recorded.

\section{Data monitoring}

Given the low risk of this intervention and integration within clinical care, we did not a convene a data monitoring committee. We developed plans for monitoring data through quarterly quality assurance assessments and conducting qualitative interviews HCPs to examine advantages and disadvantages of the programme.

\section{Outcome measures}

The number of PrEP initiation prescriptions constitute the primary outcome (see table 3). We hypothesise that the mean number of prescriptions issued is significantly higher when the clinics use the PrEP intervention versus when they do not. Secondary objectives include: (1) differences in PrEP initiation, duration of use and reasons for discontinuation based on patient's age, race/ ethnicity and sex/gender, and by clinic and HCP characteristics, (2) sustainability of the intervention during a 12-month follow-up after the stepped-wedge phase and 
Table 3 Patient-level, HCP-level and clinic-level variables

\begin{tabular}{llll}
\hline Data & Subcategory & Characteristic & Data source \\
\hline Patient level & Demographics & Age, race/ethnicity, sex/gender, primary language. & EHR \\
& HIV risk & PWID: age, enrolment in a methadone maintenance programme & Risk assessment \\
& & in last 6 months, composite injection score (inject heroin, inject &
\end{tabular}
cocaine, share cooker, share needles, visit shooting gallery).

MSM: age, no of male sex partners in past 6 months, any receptive Risk assessment anal sex with a man without a condom, no of HIV+/HIV status unknown male partners in past 6 months, any commercial sex work in past 6 months, sexually transmitted infections (STIs), methamphetamine use in past 6 months, cocaine/crack or popper use, insertive anal sex without a condom with an HIV+ or HIV status unknown man, heavy alcohol use.

Heterosexual sex: no of opposite-sex sex partners in past 6 months, sex without a condom with a woman at high risk for HIV

Risk assessment (eg, PWID) or HIV+/HIV status unknown, sex without a condom with a man at high risk for HIV (eg, PWID or bisexual male) or HIV+/ HIV status unknown, any commercial sex work in past 6 months, STIs.

\begin{tabular}{|c|c|c|c|}
\hline & $\begin{array}{l}\text { Secondary } \\
\text { Outcomes }\end{array}$ & $\begin{array}{l}\text { PrEP initiation (based on patient self-report), duration of PrEP } \\
\text { use (based on patient self-report of time of PrEP initiation to } \\
\text { discontinuation), reason for discontinuation. }\end{array}$ & EHR \\
\hline \multirow[t]{2}{*}{ HCP level } & Demographics & $\begin{array}{l}\text { Age, sex/gender, race/ethnicity, languages spoken, degree, years } \\
\text { of experience as HCP. }\end{array}$ & Baseline survey \\
\hline & Clinical care & $\begin{array}{l}\text { Provision of clinical care to individuals living with HIV }(\mathrm{Y} / \mathrm{N}) \text {, no of } \\
\text { patients living with HIV, no of patients initiated on PrEP, willingness } \\
\text { to prescribe PrEP to adolescents or adults, who in the clinic } \\
\text { provides PrEP services (such as sexual risk reduction counselling, } \\
\text { PrEP adherence counselling and laboratory tests and monitoring), } \\
\text { how confident the HCP is that they or someone in the clinic can } \\
\text { provide these services, how often the HCP assesses HIV risk } \\
\text { during patient visits, who the HCP considers prescribing PrEP to } \\
\text { and what concerns the HCP has about PrEP. }\end{array}$ & Baseline survey \\
\hline Clinic level & Characteristics & $\begin{array}{l}\text { Patient panel size, no of HCPs who have served for at least } 2 \\
\text { years, no of bilingual HCPs, ratio of ancillary staff to HCPs. }\end{array}$ & $\begin{array}{l}\text { Meeting with medical } \\
\text { director }\end{array}$ \\
\hline
\end{tabular}

EHR, electronic health record; HCP, healthcare provider; MSM, men who have sex with men; PrEP, pre-exposure prophylaxis; PWID, people who inject drugs.

(3) facilitators and barriers of PrEP delivery and experiences with the proposed PrEP intervention through qualitative interviews with HCPs.

\section{DATA ANALYSIS PLAN}

\section{Quantitative analysis plan}

Preliminary analyses and missing data

Frequency tables for all variables and measures of central tendency and variability for continuous variables will characterise the sample. In general, we will address incomplete data with maximum likelihood (ML) and multiple imputation $(\mathrm{MI})^{34}$ because they make the relatively mild assumption of a conditionally missing-at-random (MAR) data missingness mechanism. ${ }^{35}$ Auxiliary variables will be included to help meet the MAR assumption ${ }^{36} 37$ and sensitivity analyses will be conducted with weighted $\mathrm{MI}^{38}$ to assess the robustness of the MAR assumption. ${ }^{39}$ Wewill use SAS (version 9.4 or higher), ${ }^{40}$ Stata (version 16.1 or higher), and/orMplus (version 8.4 or higher) to perform analyses.

\section{Primary analysis}

To evaluate efficacy of the PrEP-OI (PrEP Coordinator + PrEP-Rx) to increase PrEP initiation prescriptions through a stepped-wedge design approach among 10 
San Francisco primary care clinical sites and to test our primary hypothesis, we will follow the approach of Hussey and Hughes, ${ }^{31}$ which is to use a mixed-models approach to estimate the effect of the intervention with a random intercept term included to reflect clustering of HCPs within clinics. To account for the effects of time, months of measurement from the stepped-wedge design will be included using a restricted cubic spline ${ }^{41}$ following the method of Amanyire et al, ${ }^{42}$ published in Lancet HIV. Alpha will be set at 0.05 for the single planned comparison of mean prescription numbers during intervention exposure to mean prescription numbers during intervention non-exposure over the stepped-wedge phase. HCPs rather than clinics will be the unit of analysis to maximise power and precision in the presence of potentially unequal numbers of HCPs per clinic. ${ }^{31}$ Summary data from local clinics suggest the number of prescriptions will be sufficiently large to treat this outcome as continuous enabling the use of a linear mixed model (LMM) to test the primary hypothesis. If the distribution of prescription numbers is sufficiently continuous but right skewed, a $\log$ transformation will be used to normalise the distribution. LMMs will be fitted to the data using SAS PROC MIXED. ${ }^{23}$ Adequacy of normality and constant variances assumptions will be assessed via histograms of model residuals and plots of predicted values by Cholesky-scaled residuals, ${ }^{43}$ respectively. However, if the outcome cannot be treated as continuous, models from the Poisson family (eg, negative binomial) will be estimated using the generalised LMMs (GLMMs) framework. GLMMs will be fitted using SAS PROC GLIMMIX with ML estimation via adaptive quadrature with a minimum of 15 integration points. ${ }^{44}$ Due to the finite number of clinics, inferences will be based on robust variance estimators with superior performance in scenarios with small numbers of clusters. ${ }^{45}$

\section{Secondary exploratory analyses}

Secondary analyses include the comparison of clinical sites randomised to the PrEP intervention versus not yet randomised, at each time point. These comparisons will maintain nominal $\alpha=0.05$ via simulation-based stepdown multiple comparison methods available in SAS PROC PLM. ${ }^{46}$ To explore differences in PrEP initiation, duration of use, and reasons for discontinuation based on patient's age, race/ethnicity, and sex/gender, and by clinic and HCP characteristics among study clinics, we will adapt the mixed models described above to incorporate patient-level outcomes (see table 3). In particular, age and sex will be considered important biological variables which may modify the effects of PrEP intervention implementation on patient-level outcomes. HCP-level and clinic-level (see table 3) covariates will also be included in these models, enabling examination of clinic-level, HCP-level and patient-level predictors' influence on PrEP prescriptions simultaneously. Patient-level outcomes are readily incorporated in the GLMM framework described above by adding an additional random intercept term to account for non-independence of patients within HCPs. The overall mixed modelling strategy will follow the same approach described above for the proposed primary analysis, leading to a unified framework which will provide a consistent analytic methodology to address both the primary and secondary quantitative aims.

\section{Qualitative analysis plan}

All interview recordings will be transcribed, and identifying information redacted. After initial review of the transcripts, two investigators will develop a codebook consisting of both a priori and emergent codes. The a priori codes will be based on the topics to be covered under the interview guide (eg, PrEP prescription practices before intervention, how practices have changed since intervention). Emergent codes will be added as needed based on interview content. After finalising the codebook, one investigator will apply the codes to each transcript, while the second investigator will review and verify the first investigator's work. Disagreements will be resolved by consensus. Following the tenets of Framework Analysis ${ }^{47}$ interview content will then be charted into a framework matrix, which will be used to interpret the data. Investigators specifically will be looking for areas of convergence and divergence across transcripts. We will seek to understand how PrEP-OI has changed PrEP prescribing and monitoring among HCPs and identify features or outcomes emerging from the intervention that have facilitated or hindered acceptance.

\section{DISCUSSION}

In this paper, we describe PrEP-OI, targeted at HCPs to increase PrEP uptake and persistence among those at risk for HIV acquisition, and the stepped-wedge study designed to evaluate its efficacy to increase PrEP prescriptions. This study builds on a pilot study showing that the intervention was feasible and acceptable for HCPs prescribing PrEP, improved their PrEP knowledge and increased ease of PrEP prescription. ${ }^{22}$

While panel management and patient navigation have been used in other chronic conditions, including Hepatitis $\mathrm{C}$ and HIV, little has been studied about their usefulness in the HIV prevention field. One study looking at PrEP navigation in a sexual health clinic in New York City noted the important services that PrEP navigators provided in moving patients along the HIV prevention continuum and allowed for necessary clinical follow-up. However, their PrEP continuum in this study ended at the provision of initial PrEP prescription, and they noted that the highest drop-off in their continuum occurred at linkage to an external PrEP provider. ${ }^{48}$

STIs clinics also face challenges in PrEP follow-up due to not having systems set up to provide continuity of care and often serving patients on a drop-in basis. Another intervention in safety-net primary care clinics in San Francisco found that patient navigation and panel management is associated with earlier PrEP initiation and greater 
completion of PrEP laboratory monitoring, which can prevent HIV infections, decrease risk of HIV drug resistance, ensure STIs are detected and treated, and may facilitate PrEP persistence. ${ }^{49} 50$ However, this was a retrospective analysis completed outside of a clinical trial. Our study aims to further the knowledge around PrEP implementation by providing a rigorous research approach to examining the impact of PrEP coordination with a web-based panel management strategy that is integrated into a primary care setting and can support patients and providers along the complete PrEP continuum from identification of potential PrEP candidates to initiation to monitoring and retention.

All of the clinics in this study are primary care clinics located in San Francisco, California. These clinics serve a wide variety of patients, including undocumented patients. Furthermore, since these clinics focused on integrated primary care, the intervention may facilitate linkage to care with their PCP when patients report acute HIV symptoms or need treatment for positive STI results. While these are unique and beneficial aspects to this study, they also create limitations on the generalisability to non-primary care clinics in other geographic locations. Additionally, PrEP-Rx is not yet integrated into the EHR used by the HCPs. We aim to explore opportunities to integrate this panel management tool with EHRs in the future. Finally, a disadvantage of the stepped wedge design of this study is that it cannot fully account for possible confounding of time with secular trends induced by outside public health interventions. However, we are not aware of any public health interventions or other phenomena that would alter the secular trend in our outcomes.

We anticipate our findings will demonstrate that with the support of a PrEP Coordinator and PrEP-Rx, primary care clinics can increase PrEP uptake and persistence among those at risk for HIV, and improve PrEP follow-up and monitoring parameters to be more closely in line with the CDC guidelines. Future studies should look into using this model to expand patient coordination services to an HIV-neutral status (ie, including people living with HIV), and if a similar intervention would be useful in non-primary care settings or with other chronic conditions.

Contributors KM and IS contributed equally to the drafting of this manuscript. $\mathrm{KM}$ served as project manager, helped develop the study protocol, and supported refinement of the PrEP-Rx tool. IS served as a PrEP Coordinator, helped develop the study protocol, and created the initial PrEP Coordination manual. AV, JW and VJ contributed to updating of the PrEP Coordination manual and carried out PrEP Coordination activities. VJ contributed to data collection, and PS, NL and TBN contributed to analysis plan. PS conceived of the study, served as principal investigator, and provided final approval of the version to publish. PS, TBN, HS, AL, WS and MOJ developed the design and measures. All authors were involved in the revision of the draft manuscript and approved the submitted version.

Funding This work was supported by the National Institute of Nursing Research grant number R01NR017573.

Competing interests AL has received an investigator sponsored research grant from Gilead Sciences, and has led studies in which Gilead has donated study drug.
Patient and public involvement Patients and/or the public were involved in the design, or conduct, or reporting, or dissemination plans of this research. Refer to the Methods section for further details.

Patient consent for publication Not required.

Provenance and peer review Not commissioned; peer reviewed for ethical and funding approval prior to submission.

Open access This is an open access article distributed in accordance with the Creative Commons Attribution Non Commercial (CC BY-NC 4.0) license, which permits others to distribute, remix, adapt, build upon this work non-commercially, and license their derivative works on different terms, provided the original work is properly cited, appropriate credit is given, any changes made indicated, and the use is non-commercial. See: http://creativecommons.org/licenses/by-nc/4.0/.

ORCID iDs

Kristin Ming http://orcid.org/0000-0002-0688-9002

Parya Saberi http://orcid.org/0000-0002-3793-5112

\section{REFERENCES}

1 Grant RM, Lama JR, Anderson PL, et al. Preexposure chemoprophylaxis for HIV prevention in men who have sex with men. N Engl J Med 2010;363:2587-99.

2 Thigpen MC, Kebaabetswe PM, Paxton LA, et al. Antiretroviral preexposure prophylaxis for heterosexual HIV transmission in Botswana. N Engl J Med 2012;367:423-34.

3 Baeten JM, Donnell D, Ndase P, et al. Antiretroviral prophylaxis for HIV prevention in heterosexual men and women. N Engl J Med 2012;367:399-410.

4 Choopanya K, Martin M, Suntharasamai P, et al. Antiretroviral prophylaxis for HIV infection in injecting drug users in Bangkok, Thailand (the Bangkok tenofovir study): a randomised, double-blind, placebo-controlled phase 3 trial. Lancet 2013;381:2083-90.

5 Molina J-M, Capitant C, Spire B, et al. On-Demand preexposure prophylaxis in men at high risk for HIV-1 infection. N Engl J Med 2015;373:2237-46.

6 McCormack S, Dunn DT, Desai M, et al. Pre-exposure prophylaxis to prevent the acquisition of HIV-1 infection (PROUD): effectiveness results from the pilot phase of a pragmatic open-label randomised trial. Lancet 2016;387:53-60.

7 Baeten JM, Heffron R, Kidoguchi L, et al. Integrated delivery of antiretroviral treatment and pre-exposure prophylaxis to HIV-1Serodiscordant couples: a prospective implementation study in Kenya and Uganda. PLoS Med 2016;13:e1002099.

8 Hosek SG, Rudy B, Landovitz R, et al. An HIV preexposure prophylaxis demonstration project and safety study for young MSM. $J$ Acquir Immune Defic Syndr 2017;74:21-9.

9 Liu AY, Cohen SE, Vittinghoff E, et al. Preexposure prophylaxis for HIV infection integrated with Municipal- and community-based sexual health services. JAMA Intern Med 2016;176:75-84.

10 Marcus JL, Hurley LB, Nguyen DP, et al. Redefining human immunodeficiency virus (HIV) preexposure prophylaxis failures. Clin Infect Dis 2017;65:1768-9.

11 Krakower D, Ware N, Mitty JA, et al. HIV providers' perceived barriers and facilitators to implementing pre-exposure prophylaxis in care settings: a qualitative study. AIDS Behav 2014;18:1712-21.

12 Krakower DS, Mayer KH. Pre-Exposure prophylaxis to prevent HIV infection: current status, future opportunities and challenges. Drugs 2015;75:243-51.

13 Karris MY, Beekmann SE, Mehta SR, et al. Are we prepped for preexposure prophylaxis (PrEP)? Provider opinions on the realworld use of PrEP in the United States and Canada. Clin Infect Dis 2014;58:704-12.

14 United States - PrEPWatch [Internet], 2020. Available: https://www. prepwatch.org/country/united-states/

15 Smith DK, Van-Handel M, Wolitski RJ, et al. Vital signs: estimated percentages and numbers of adults with indications for preexposure prophylaxis to prevent HIV acquisition - United States, 2015. Morb Mortal Wkly Rep 2015;56:364-71.

16 Marcus JL, Volk JE, Pinder J, et al. Successful implementation of HIV preexposure prophylaxis: lessons learned from three clinical settings. Curr HIVIAIDS Rep 2016;13:116-24.

17 Blumenthal J, Jain S, Krakower D, et al. Knowledge is power! Increased provider knowledge scores regarding pre-exposure prophylaxis (PrEP) are associated with higher rates of PrEP prescription and future intent to prescribe PrEP. AIDS Behav 2015;19:802-10. 
18 Cohen SE, Liu AY, Bernstein KT, et al. Preparing for HIV pre-exposure prophylaxis: lessons learned from post-exposure prophylaxis. Am J Prev Med 2013;44:S80-5.

19 Smith DK, Mendoza MCB, Stryker JE, et al. PrEP awareness and attitudes in a national survey of primary care clinicians in the United States, 2009-2015. PLoS One 2016;11:e0156592.

20 Liu A, Cohen S, Follansbee S, et al. Early experiences implementing pre-exposure prophylaxis (PrEP) for HIV prevention in San Francisco. PLoS Med 2014;11:e1001613.

21 Kelley CF, Kahle E, Siegler A, et al. Applying a PrEP continuum of care for men who have sex with men in Atlanta, Georgia. Clin Infect Dis 2015;61:1590-7.

22 Saberi P, Berrean B, Thomas S, et al. A simple pre-exposure prophylaxis (PrEP) optimization intervention for health care providers prescribing PrEP: pilot study. JMIR Form Res 2018;2. doi:10.2196/ formative.8623. [Epub ahead of print: 16 Jan 2018].

23 Centers for Disease Control and Prevention. Preexposure prophylaxis for the prevention of HIV infection in the United States-2017 Update: a clinical practice guideline [Internet. US Public Health Service, 2017. https://www.cdc.gov/hiv/pdf/risk/prep/cdc-hiv-prepguidelines-2017.pdf

24 Hare CB. Abstract 104LB. Conference on Reteroviruses and Opportunistic Infections, Seattle, 2019.

25 Smith DK, Pan Y, Rose CE, et al. A brief screening tool to assess the risk of contracting HIV infection among active injection drug users. $J$ Addict Med 2015;9:226-32.

26 Lu M, Safren SA, Skolnik PR, et al. Optimal recall period and response task for self-reported HIV medication adherence. AIDS Behav 2008;12:86-94.

27 Giordano TP, Guzman D, Clark R, et al. Measuring adherence to antiretroviral therapy in a diverse population using a visual analogue scale. HIV Clin Trials 2004;5:74-9.

28 Hayes RJ, Moulton LH. Cluster randomised trials. cluster randomised trials. Boca Raton, FL: Chapman and Hall, 2009.

29 Hemming K, Girling A. A menu-driven facility for power and detectable-difference calculations in Stepped-Wedge clusterrandomized trials. Stata J 2014;14:363-80.

30 Crosby RA, Rothenberg R. In STI interventions, size matters. Sex Transm Infect 2004;80:82-5.

31 Hussey MA, Hughes JP. Design and analysis of stepped wedge cluster randomized trials. Contemp Clin Trials 2007;28:182-91.

32 Stare A. Agile project management - a future approach to the management of projects? Dyn Relationships Manag J 2013;2:43-53.

33 Coyne IT. Sampling in qualitative research. Purposeful and theoretical sampling; merging or clear boundaries? J Adv Nurs 1997;26:623-30.

34 Schafer JL, Graham JW. Missing data: our view of the state of the art. Psychol Methods 2002;7:147-77.
35 Little RJA, Rubin DB. Statistical analysis with missing data. New York: John Wiley \& Sons, 2014.

36 Collins LM, Schafer JL, Kam C-M. A comparison of inclusive and restrictive strategies in modern missing data procedures. Psychol Methods 2001;6:330-51.

37 Graham JW. Adding missing-data-relevant variables to FIML-based structural equation models. Struct Equ Model 2003;10:80-100.

38 Carpenter JR, Kenward MG, White IR. Sensitivity analysis after multiple imputation under missing at random: a weighting approach. Stat Methods Med Res 2007;16:259-75.

39 Hedeker D, Gibbons RD. Application of random-effects patternmixture models for missing data in longitudinal studies. Psychol Methods 1997;2:64-78.

40 SAS Institute. SAS on-line doc, version 9.0. NC: SAS Institute, Inc, 2002.

41 Harrell FE. Regression modeling strategies. New York: SpringerVerlag, 2015.

42 Amanyire G, Semitala FC, Namusobya J, et al. Effects of a multicomponent intervention to streamline initiation of antiretroviral therapy in Africa: a stepped-wedge cluster-randomised trial. Lancet HIV 2016;3:e539-48.

43 Petroll AE, Mosack KE. Physician awareness of sexual orientation and preventive health recommendations to men who have sex with men. Sex Transm Dis 2011;38:63-7.

44 Bernstein KT, Liu K-L, Begier EM, et al. Same-Sex attraction disclosure to health care providers among New York City men who have sex with men: implications for HIV testing approaches. Arch Intern Med 2008;168:1458-64.

45 Morel JG, Bokossa MC, Neerchal NK. Small sample correction for the variance of GEE estimators. Biometrical J 2003;45:305-409.

46 Westfall PH, Young SS. Resampling-Based multiple testing: examples and methods for $p$-Value adjustment. New York: John Wiley \& Sons, 1993.

47 Gale NK, Heath G, Cameron E, et al. Using the framework method for the analysis of qualitative data in multi-disciplinary health research. BMC Med Res Methodol 2013;13:117.

48 Pathela P, Jamison K, Blank S, et al. The HIV Pre-exposure prophylaxis (PrEP) cascade at NYC sexual health clinics. JAIDS J Acquir Immune Defic Syndr 2020;83:357-64.

49 Spinelli MA, Scott HM, Vittinghoff E, et al. A panel management and patient navigation intervention is associated with earlier prep initiation in a safety-net primary care health system. J Acquir Immune Defic Syndr 2018;79:347-51.

50 Spinelli MA, Scott HM, Vittinghoff E, et al. Provider adherence to preexposure prophylaxis monitoring guidelines in a large primary care network. Open Forum Infect Dis 2018;5:ofy099. 